\title{
Leukocytic picture in pediatric Coronavirus Disease-19 infection
}

\author{
Pranoy Dey, Rhitwika Das \\ From Professor, Post Graduate Trainee, Department of Paediatrics, Assam Medical College and Hospital, Dibrugarh, Assam, India
}

\begin{abstract}
Background: Coronavirus is a large family of enveloped single-stranded zoonotic RNA virus, causes a wide spectrum of disease including Coronavirus disease-2019 (COVID-19). It is a systemic disease where immunological response has been cited as the most important parameter for both COVID-19 related death as well recoveries. Among all the blood parameters, leukocytes play the most important role in mounting an immune response. COVID-19 being a novel virus has enough literature on its leukocytic picture in adult population which is being lacking in pediatrics study. Aim: The aim of the study was to study the leukocyte picture of COVID-19 positive pediatric populations admitted in Assam Medical College and Hospital. Methods: An observational study was conducted on COVID-19 positive pediatrics patients (Age $>1$ month-12 years) as detected by rapid antigen test/reverse transcription-polymerase chain reaction and whose blood samples could be sent for investigations. Patient's status and leukocytic picture at the time of admission were studied. They were followed up till discharge. Results: A total 54 COVID-19 positive patients were enrolled in the study. The mean age group was $8 \pm 2$ years and gender was male predominant. Out of 54 patients enrolled in the study, 38.89\% had lymphocytopenia, $25.9 \%$ neutropenia, $24 \%$ normal blood picture, and $12.96 \%$ had leukocytosis. Four patients expired and 50 patients were discharged. Neutropenia was found in $100 \%$ of the mortalities. Conclusion: Lymphocytopenia is the most commonly found deranged blood parameter. Neutropenia when found in COVID-19 positive pediatrics population, signify poor prognosis.
\end{abstract}

Key words: Coronavirus disease-19, Leukocytic picture, Lymphocytopenia, Neutropenia

$\mathrm{C}$ oronaviruses are a large family of zoonotic viruses [1], causing a wide spectrum of diseases ranging from mild common flu to severe acute respiratory syndrome (SARS$\mathrm{CoV}-1)$. It can rapidly mutate and recombine leading to novel coronavirus formation that spreads among human population forming a new strain [1]. In humans, they mostly cause respiratory tract and gastrointestinal tract related symptoms [1,2], yet, its systemic manifestations cannot be denied.

SARS-CoV-2 was first identified in a group of patients with respiratory tract symptoms in Wuhan Province in China at the end of December, 2019 [1,2] and was officially named as Coronavirus disease-2019 (COVID-19) by the World Health Organization (WHO) on February 2, 2020 [3-5]. It was declared as a pandemic disease by the WHO on March 11, 2020 [3].

Inflammation plays a key role in the development of COVID-19 and is likely an important factor in its prognosis [6-8]. Immunological response is both an armor as well as a threat in COVID-19 infections. Quantitative and qualitative changes in peripheral blood cells have been described in adults with COVID-19 [6-8]. Among all the blood parameters, namely,

\section{Access this article online}

Received - 24 March 2021

Initial Review - 19 April 2021

Accepted - 15 July 2021

DOI: 10.32677/IJCH.2021.v08.i08.2980 erythrocytes, leukocytes, and platelets; leukocytes play an important role in mounting an immune response in our body. Neutrophils constitute the majority of the leukocytes and are primarily responsible for activating the immune system by migrating from the venous system. Free oxygen radicals that can damage the nuclear material of the cell are thereby released. Viral antigens are exposed and cell-specific and humoral immunities are stimulated by an antibody-dependent cell-mediated response. There is a growing interaction with molecules such as vascular endothelial growth factor (VEGF), interleukin-6, interleukin-8, tumor necrosis factor-alpha (TNF- $\alpha$ ), interferon-gamma, and granulocyte colony-stimulating factor. VEGF-A and VEGF-C are particularly found to be notorious in the novel coronavirus [9]. $\mathrm{T}$ helper cells induce the production of cytokines such as interleukin-17 through the nuclear factor kappa-light-chainenhancer of activated B cells (NF-kB) signaling pathway, leading to increased aggregation of monocytes. SARS-CoV-2 infects the circulating immune cells and increases apoptosis of lymphocytes, leading to lymphopenia. A lower ratio of circulating lymphocytes to monocytes predicts severe and extremely severe COVID-19 as the clearance of the virus is delayed due to lymphopenia and also due to a decrease in $\mathrm{CD} 4+\mathrm{T}$ cells, as evident in studies done in adults [9]. IL6, IL8, IL17, interferon gamma, and TNF-alpha

Correspondence to: Rhitwika Das, Department of Paediatrics, Assam Medical College and Hospital, Dibrugarh, Assam, India. E-mail: rhitwika27@gmail.com

(C) 2021 Creative Commons Attribution-NonCommercial 4.0 International License (CC BY-NC-ND 4.0). 
are some of the investigations scrutinized to be of great help as a prognostic marker in COVID-19 in countries with resource and healthcare abundance. However, in a resource limited country with barely accessible healthcare facilities to modern care of treatment, routine investigation of blood comprising differential leukocytic count can act as a boon. Therefore, through this study we aimed to study the leukocytic picture and lymphocyte: monocyte ratio as a prognostic marker in COVID-19 affected pediatric population.

The objective of this study was to record the leukocytic picture in COVID-19 positive pediatric population as well as to determine the prognosis of COVID-19 using lymphocyte: monocyte ratio as an indicator.

\section{METHODS}

It was an observational study conducted in Assam Medical College and Hospital after getting clearance from the Institutional Ethical Committee. All pediatric patients (age $>1$ month-12 years), found positive for COVID-19 and whose blood samples could be sent for investigations were included in the study. Patients expiring within three hours of hospital admission and age $<1$ month or $>12$ years, were excluded from the study.

Patients were tested for COVID-19 by quantitative real-time reverse transcription-polymerase chain reaction (qRT-PCR) from nasal and oropharyngeal swab samples or by rapid antigen test (RAT) for COVID-19 from nasal swab. COVID-19 positive patients were further classified into mild, moderate, and severe categories [10]. Complete blood count (CBC) and peripheral smear (Siemens ADVIA 2120i hematology analyzer with auto slide) were done on admission. CBC values were recorded and assessed according to age-specific intervals [11]. Peripheral blood smears were evaluated blindly, and differential counts were formulated by manual counting. Lymphocyte: monocyte ratio was calculated using MS Excel. They were followed up till discharge. The data were collected and tabulated on MS Excel. Values were expressed as mean \pm Standard deviation.

\section{RESULTS}

Total 57 patients (age $>1$ month-12 years), were found positive for COVID-19 by RAT/RT-PCR. Out of them, three succumbed to death within $2 \mathrm{~h}$ of hospital admission and hence were not included in the study as they did not fulfill the eligibility criteria. Thus, 54 patients were enrolled in our study.

Out of 54 patients, ten patients had severe and 44 patients had moderate COVID-19 infections. It was found that lymphocyte: monocyte ratio in the ten severe cases, were $<1.5$ and in the moderate cases were ranged 1.5-3. The leukocytic picture had lymphocytopenia as the predominant deranged blood parameter (34.4\%), followed by neutropenia $(25.5 \%)$. It was followed by a normal blood picture in $24 \%$ of the population while $12 \%$ population had leukocytosis as the blood picture. The patients were followed up till discharge. Out of the 54 patients, 92\% (50) could be discharged successfully, whereas $8 \%$ (4) patients succumbed to death. Among the expiries, neutropenia was found in $100 \%$ cases of mortality. Few more notable parameters are mentioned below:

- Demographic profile: Mean age group- $8 \pm 2$ years and the male: female ratio-1.6:1

- Clinical profile: Shown in Fig. 1

- Leukocytic picture: Shown in Fig. 2

- Leukocytic parameter in expired patients: Shown in Fig. 3.

\section{DISCUSSION}

It can be emphasized from the study that lymphocytopenia is the most common deranged leukocytic parameter in

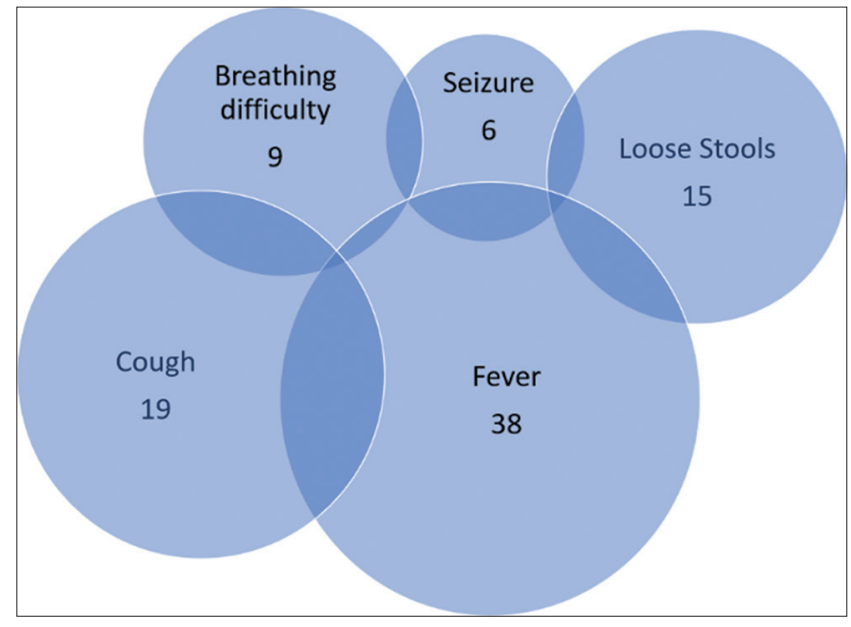

Figure 1: Clinical profile of the enrolled patients

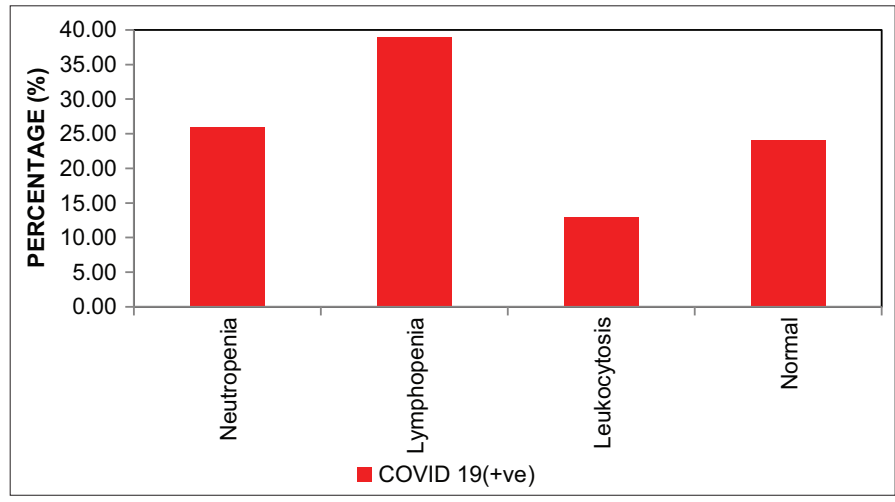

Figure 2: Bar diagram showing leukocytic picture in COVID-19

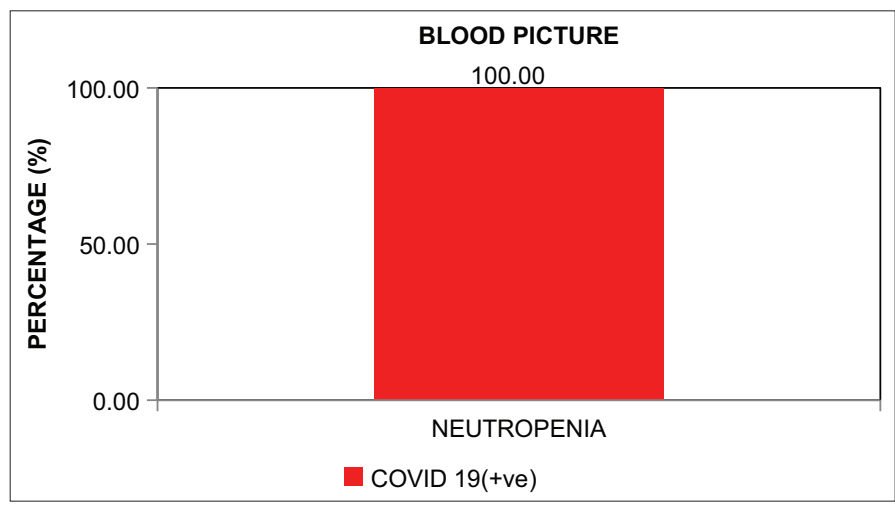

Figure 3: Bar diagram showing leukocytic picture in COVID-19 positive mortality 
pediatric age group in COVID-19 infection. In adults, the most common hematological findings of COVID-19 also includes lymphocytopenia, observed by Li et al. [1,2] and supported by Sumeyye et al. [11]. Thus, there is no vast difference in the immunological response mounted by the leukocytes among the pediatric and adult age groups in terms of COVID-19 infection. The studies done by Pari et al., Oiu et al., Xia et al. done in Italy and two provinces of China found lymphocytopenia as the most common deranged blood parameter [12], which was in accord with our study. Another study done by Chao et al. [12] in a Tertiary Centre Children Hospital in New York City found leukocytosis as the most predominant deranged blood parameter and their findings were found to be contradictory to ours.

Both Lymphocytopenia and neutropenia were detected by Nesi et al. in their study [12] but the neutropenia observed in their study was not related to any mortality as observed in our study which had neutropenia in all $100 \%$ cases of mortality. Thus, it can be deduced that neutropenia in COVID-19 infection signifies poor prognosis.

We found that all the severe COVID-19 cases had significantly low lymphocyte: monocyte ratio which was at par with the findings by Shivakumar et al. in adult populations [9].

Leukocytic picture, as provided by the $\mathrm{CBC}$ report is an easily available, cost effective tool that can be used for both screening and prognosis analysis of COVID-19 infection. It is widely available even in the remotest part of our country.

However, our study had certain limitations as well. The leukocytic picture could not be compared with populations with COVID-19 negative status due to logistic constraints. Throat swab and blood cultures of all the cases could not be taken due to resource crisis. Computed tomography values correspondence of COVID-19 infection as detected by RT-PCR could not be read as many of the cases were RAT positive. For these cases, RT-PCR was not done at the point of admissions to preserve the already limited resources.

\section{CONCLUSION}

COVID-19 is a novel virus with prominent hematological findings. Leukocytic picture is a convenient and easily available cheap blood analysis which would help in screening as well as indicating the prognosis. Current study reports that the leukocytic picture in COVID-19 infection is similar in both adults and children. Lymphocytopenia and neutrophilia are the most common deranged blood parameter which would be helpful in screening purpose for COVID-19 infection. Neutropenia and low lymphocyte: monocyte ratio, signify severe COVID-19 infection, it can thereby guide to make appropriate clinical decisions. With the third wave of COVID-19 infection at the verge of being unfold, it is imperative for us to channel the available resources to decrease the severity and mortality of infection. Evaluation of leukocytic picture can act as a boon in resource limited countries during the third wave of COVID-19 infection

\section{REFERENCES}

1. Li Q, Guan X, Wu P, Wang X, Zhou L, Tong Y, et al. Early transmission dynamics in Wuhan, China, of novel coronavirus-infected pneumonia. N Engl J Med 2020;382:1199-207.

2. Pal M, Berhanu G, Desalegn C, Kandi V. Severe acute respiratory syndrome coronavirus-2 (SARS-CoV-2): An update. Cureus 2020;12:e7423.

3. World Health Organization Press Conference. The World Health Organization (WHO) Has Officially Named the Disease Caused by the Novel Coronavirus as COVID-19. Geneva: World Health Organization; 2020.

4. Chan JF, Yuan S, Kok KH, To KK, Chu H, Yang J, et al. A familial cluster of pneumonia associated with the 2019 novel coronavirus indicating person-toperson transmission: A study of a family cluster. Lancet 2020;395:514-23.

5. Zhu N, Zhang D, Wang W, Li X, Yang B, Song J, et al. China novel coronavirus investigating and research team. A novel coronavirus from patients with pneumonia in China, 2019. N Engl J Med 2020;382:727-33.

6. Wang Y, Ju M, Chen C, Yang D, Hou D, Tang X, et al. Neutrophil-tolymphocyte ratio as a prognostic marker in acute respiratory distress syndrome patients: A retrospective study. J Thor Dis 2018;10:273-82.

7. Famous KR, Delucchi K, Ware LB, Kangelaris KN, Liu KD, Thompson BT, et al. ARDS network acute respiratory distress syndrome subphenotypes respond differently to randomized fluid management strategy. Am J Resp Crit Care Med 2017;195:331-8.

8. Summers C, Singh NR, White JF, Mackenzie IM, Johnston A, Solanki C, et al. Pulmonary retention of primed neutrophils: A novel protective host response, which is impaired in the acute respiratory distress syndrome. Thorax 2014;69:623-9.

9. Shivakumar BG, Gosavi S, Rao AA, Shastry S, Raj SC, Sharma A, et al. Neutrophil-to-lymphocyte, lymphocyte-to-monocyte, and plateletto-lymphocyte ratios: Prognostic significance in COVID-19. Cureus 2021;13:e12622.

10. Lu X, Zhang L, Du H, Zhang J, Li YY, Qu J, et al. SARS-CoV-2 infection in children. N Engl J Med 2020;382:1663-5.

11. Sun Y, Koh V, Marimuthu K, Ng OT, Young B, Vasoo S, et al. Epidemiological and clinical predictors of COVID-19. Clin Infect Dis 2020;71:786-92.

12. Kazancioglu S, Bastug A, Ozbay BO, Kemirtlek N, Bodur H. The role of haematological parameters in patients with COVID-19 and influenza virus infection. Epidemiol Infect 2020;148:e272.

Funding: None; Conflicts of Interest: None Stated.

How to cite this article: Dey $\mathrm{P}$, Das R. Leukocytic picture in pediatric Coronavirus Disease-19 infection. Indian J Child Health. 2021; 8(8):277-279. 